\title{
A novel cannula for uniform mixing of liquid substances
}

\author{
Seung Jun Kim${ }^{1}$, Seung Min Kim², \\ Cheol Keun Kim², Dong In Jo', \\ Soon Heum Kim ${ }^{2}$ \\ ${ }^{1}$ The Iron Plastic Surgery, Seoul; \\ ${ }^{2}$ Department of Plastic and \\ Reconstructive Surgery, Konkuk \\ University Chungju Hospital, Konkuk \\ University School of Medicine, Chungju, \\ Korea
}

\begin{abstract}
Uniform mixing of liquid substances is difficult to attain. Recently, mixtures of fat and platelet-rich plasma (PRP) have been used extensively in autologous fat transfer. Although various methods have been utilized to mix the fat and PRP involved in this procedure, these methods still carry disadvantages in the time taken and the potential for cell damage, and it is questionable whether effective mixing can occur using these techniques. To facilitate mixing that is as uniform as possible, the authors of this study invented a special cannula. Use of the conventional luer-lock-to-luer-lock connector method has been found to cause cell damage and makes it difficult to achieve uniform mixing, while random injection-type mixing has been shown to have limited accuracy and effectiveness. We expect that the newly designed cannula will solve the problem detailed above.
\end{abstract}

Keywords Cannula / Mechanical stress / Methods / Solutions / Uniform mixing

\section{INTRODUCTION}

Autologous fat transfer was first reported by Neuber in 1893 [1]. Ever since the Coleman technique [2] was introduced in 1997, consistent operative results have been obtained using that method, and autologous fat has been settled upon as a reliable natural filler. Autologous fat tissue has become a clinical reality for facial augmentation, as it is biocompatible, versatile, natural in appearance, non-immunogenic, inexpensive, and readily obtainable with low donor site morbidity [3]. However, the viability of grafted fat has been reported to be highly variable, in the range of $30 \%$ to $80 \%$ depending on the study, and is the subject of ongoing debate [4,5].

The reasons for this variability include differences in donor site preparation techniques, harvesting methods, instruments used, grafting and injection techniques, graft interval, and methods of analysis. These variations have made it difficult to compare studies $[6,7]$.

Received: Jun 10, 2019 Revised: Sep 2, 2019 Accepted: Sep 3, 2019 Correspondence: Soon Heum Kim Department of Plastic and Reconstructive Surgery, Konkuk University Chungju Hospital, Konkuk University School of Medicine, 82 Gugwon-daero, Chungju 27376, Korea Tel: +82-43-840-8860, Fax: +82-43-840-9862, E-mail: plastic7@kku.ac.kr

Copyright @ 2019 The Korean Society for Aesthetic Plastic Surgery.

This is an Open Access article distributed under the terms of the Creative Commons Attribution Non-Commercial License (http://creativecommons.org/licenses/by-nc/4.0/) which permits unrestricted non-commercial use, distribution, and reproduction in any medium, provided the original work is properly cited. www.e-aaps.org
Recently, increased attention has been focused on the co-application of platelet-rich plasma (PRP) and fat grafts. Several clinical cases have been reported in which the combination of fat grafting and PRP has been used to improve wound healing [8]. PRP has been shown to increase the fat survival rate and promote stem cell proliferation and differentiation in vitro [9]. Until now, practitioners of this technique have typically injected PRP to the recipient site separately from the fat cells or used luer-lock-to-luer-lock connectors to mix PRP with harvested fat (Fig. 1) [10]. However, mixing using a connector causes repeated trauma to adipocytes, and PRP also may not mix uniformly when this method is used. Based on the information above, the authors of this study have invented a new cannula to facilitate effective and safe mixing.

\section{IDEA}

Nessie is the imaginary monster who, according to legend, lives in the Loch Ness lake in Scotland, Inverness. In 1975, an American lawyer came forth with a picture that appeared to show a dinosaur with a long neck. The lawyer said that the photo had been taken at Loch Ness, contributing to the legend. We devised a Nessie cannula based on the mythical appearance of Nessie's curved long neck.

The Nessie cannula consists of a straight head and shaft. The length of the head is $1 \mathrm{~cm}$, and the length of the shaft is $10 \mathrm{~cm}$. The bend of the Nessie cannula measures $30^{\circ}$ and is designed to fit the diameter of a luer lock syringe. The vertical distance from the long axis to the head tip is $0.5 \mathrm{~cm}$. The cannula has three $1 \mathrm{~mm}$-diame- 


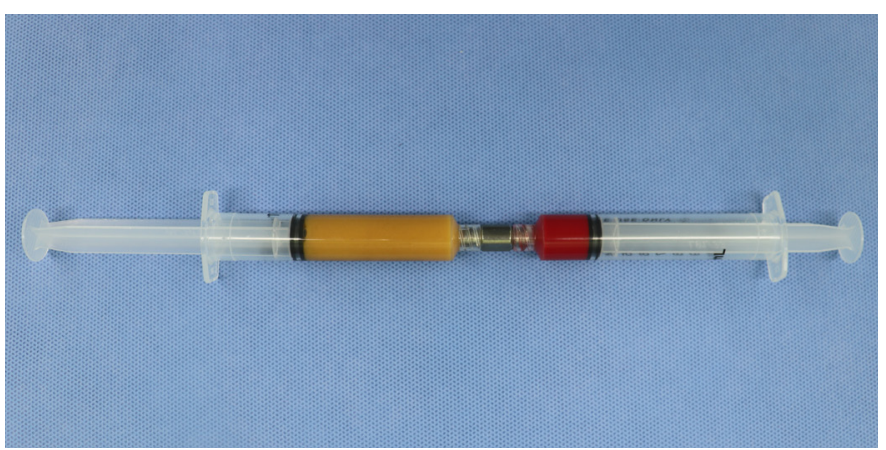

Fig. 1. Photograph illustrating a conventional luer-lock-to-luer-lock connector method for mixing liquid substances.
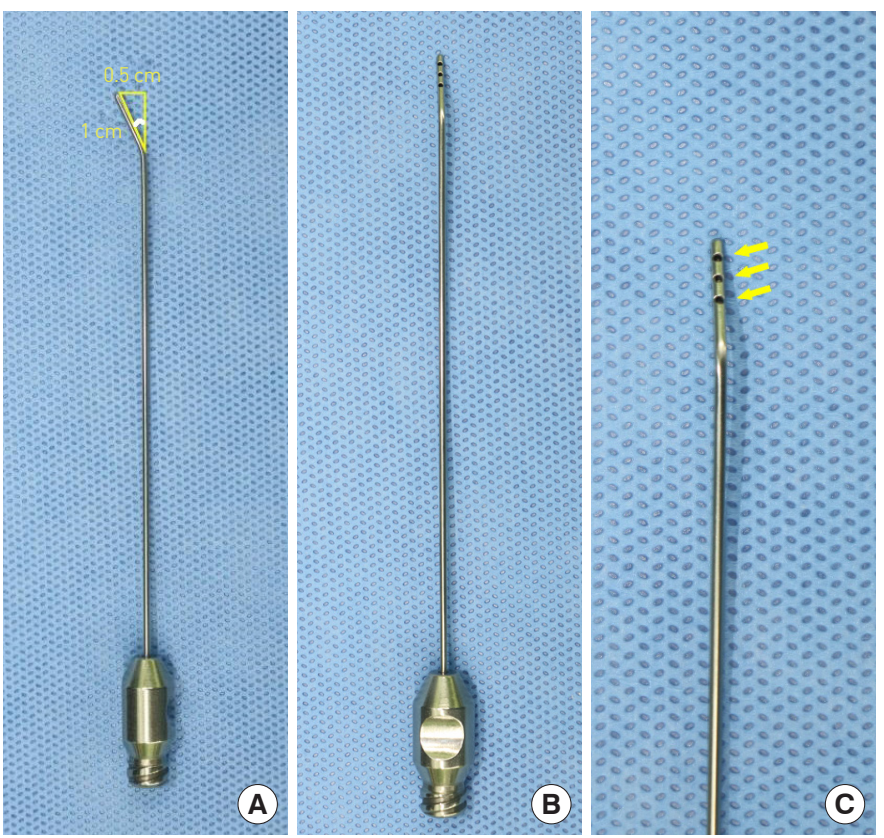

Fig. 2. Photographs of the Nessie cannula. (A, B) The Nessie cannula consists of a straight head and shaft. The length of the head is $1 \mathrm{~cm}$, and the length of the shaft is $10 \mathrm{~cm}$. The bend of the Nessie cannula has an angle of $30^{\circ}$ (white curve). The vertical distance from the bend to the tip of the head is $0.5 \mathrm{~cm}$. (C) The cannula has three $1-\mathrm{mm}$-diameter pores aligned in the head (yellow arrows).

ter pores aligned in the head (Fig. 2).

In the method we devised, a luer lock syringe containing collected PRP and a separate luer lock syringe containing harvested fat are prepared. The Nessie cannula is connected to the luer lock syringe containing the PRP. Then, the Nessie cannula is inserted into the luer lock syringe containing the purified fat and is extended toward the bottom of the syringe. The bend of the cannula is placed in the center of the syringe, and the PRP is gently injected. Concurrently, the luer lock syringe containing the purified fat is gradually turned away from the cannula (for a demonstration of this technique, Supplemental Digital Content 1). Compared to the conven-
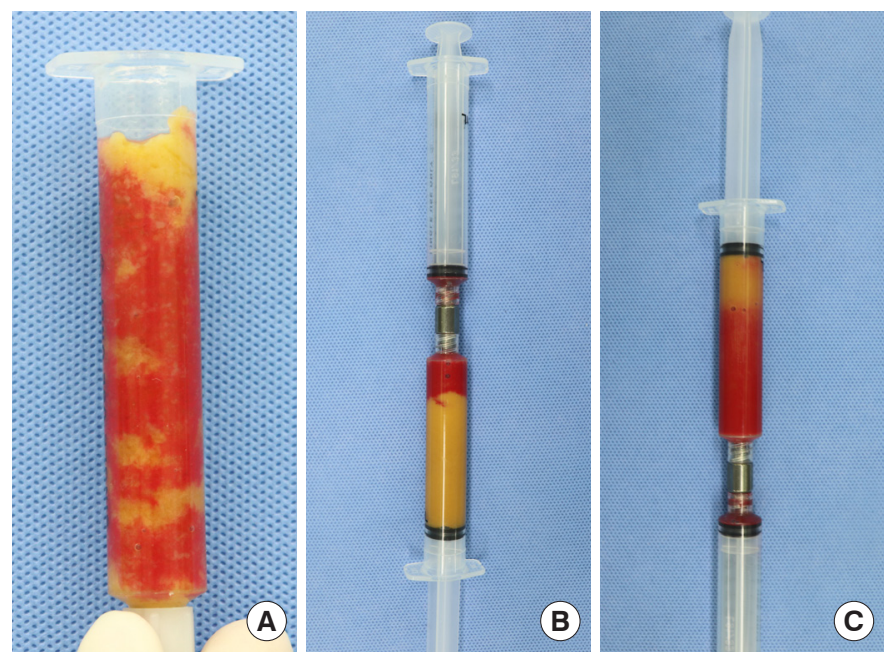

Fig. 3. A comparison of techniques used to mix purified fat with red paint diluted to the density of platelet-rich plasma. (A) The new cannula method. The spiral pattern shows a relatively uniform mixed state extending from the center to the periphery. $(B, C)$ The conventional luer-lock-to-luer-lock connector method. The liquid substances show a tendency to display en bloc distribution to a specific area.

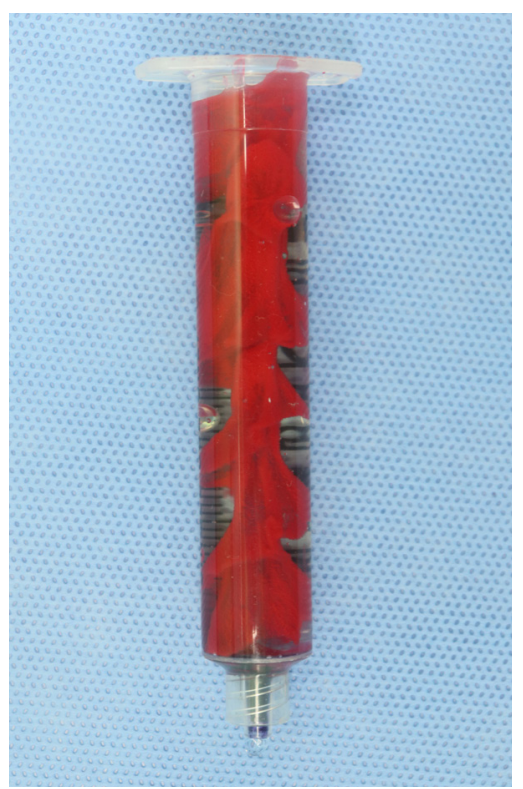

Fig. 4. The red paint diluted to the density of platelet-rich plasma was uniformly spirally distributed within the ultrasound transmission gel (Ecosonic).

tional luer-lock-to-luer-lock connector method, the cannula method yielded contents that were more uniformly mixed (Fig. 3).

As an experimental model to visualize the use of this technique, a luer lock syringe containing an ultrasound transmission gel (Ecosonic; Sanipia, Gimje, Korea) and a luer lock syringe containing red paint diluted to the density of PRP were prepared, and the con- 
tents were mixed according to the method detailed above. The paint was spirally distributed and was effectively and uniformly mixed (Fig. 4).

\section{DISCUSSION}

To improve the survival rate of transplanted fat, many studies on cannula diameter, type of cannula, donor site preparation techniques, fat grafting and injection techniques, and grafting interval have been conducted $[6,7,11]$. However, there is still no standardized method of fat transplantation that is accepted by most surgeons.

As fat transplantation has evolved, major improvements have also been made to the techniques and factors listed above. In addition, methods have been developed to improve fat cell survival by incorporating new additives, such as insulin, vascular endothelial growth factor (VEGF), and PRP $[10,12,13]$. Among these methods, autologous PRP-assisted fat grafting is emerging as an attractive option.

PRP is a concentration of autologous human platelets (three to five times higher than the baseline platelet count) in a small volume of plasma. Platelets act through the degranulation of $\alpha$-granules containing synthesized growth factors, such as platelet-derived growth factors, transforming growth factor-beta, insulin-like growth factor, VEGF, and endothelial growth factor. These factors bind to the surfaces of cell membranes to stimulate hemostasis and normal healing by promoting processes that include the activation of angiogenesis, cell proliferation, cell differentiation, and new matrix formation for tissue repair. PRP stimulates angiogenic processes and the proliferation of adipose-derived stem cells [3].

Using the conventional luer-lock-to-luer-lock connector method can cause cell damage. When abdominal lipoaspiration was performed at high pressure $(760 \mathrm{mmHg})$ and low pressure $(250 \mathrm{mmHg})$, the adipocyte count was $47 \%$ higher at low pressure than at high pressure, and cell viability was significantly higher at day 7 with low-pressure than with high-pressure aspiration [14]. Using a syringe pressure calculator, we saw that a pressure of $748 \mathrm{mmHg}$ is generated by the minimum force required to produce a full vacuum using a 10-mL BD syringe as the plunger force [15]. Therefore, mixing using connectors can reduce cell viability by causing repeated trauma to fat cells.

Furthermore, it is not known whether the PRP is evenly distributed in the fat after mixing. Additionally, it is often necessary to perform mixed injections of liquid contents other than PRP, such as hyaluronic acid (HA) plus PRP, HA plus filler, HA plus triamcinolone, filler plus filler, and fat plus PRP. The cannula that we devised helps to mix substances as uniformly as possible with as little damage as possible, facilitating the proper functioning of all injected substances after injection. In particular, for PRP plus fat, using the cannula can reduce trauma in the mixing process, and it can improve the survival of adipocytes by maximizing contact between
PRP and the harvested fat, thereby improving the surgical outcome.

The advantages of the Nessie cannula are as follows. The first advantage is that uniform mixing is achieved. The cannula's head has three pores, allowing it to mix up to the periphery of the fatcontaining syringe, and the cannula has an angle between the head and the shaft that ensures that its contents can be injected evenly throughout the syringe. By turning the fat-containing syringe with one's free hand, it is possible to adjust the amount and position of the mixing, ensuring that the liquid substances are mixed as uniformly as possible. Random injection of PRP into the prepared fat cannot be expected to achieve uniform mixing. A second advantage is that atraumatic mixing is achieved. In the conventional luerlock-to-luer-lock connector method, adipocytes move through a narrow space several times while being subjected to mechanical force, resulting in increased trauma and cell death and reduced engraftment rate. Third, this method is simple, fast, and inexpensive. The cannula is manufactured to fit the luer lock syringe and securely connect to it. On a practical level, we have found this method to be extremely simple to perform, meaning that anyone trained in the method can apply it easily.

The Nessie cannula is effective in uniformly mixing liquid or gel-like substances together. For instance, it can be easily applied to mix HA with PRP, HA with filler, HA with triamcinolone, filler with filler, or fat with PRP. Mixture of the above substances using alternative mixing techniques can lead to problems, such as an increased number of injections required, an increased risk of infection, and uneven injection. We have obtained a Korean patent for this cannula (Registration No. 40-1466545-0000). The effectiveness of uniform mixing using the Nessie cannula in clinical cases requires further study, which we plan to carry out.

\section{NOTES}

\section{Conflict of interest}

No potential conflict of interest relevant to this article was reported.

\section{Ethical approval}

The study was performed in accordance with the principles of the Declaration of Helsinki.

\section{ORCID}

Seung Jun Kim

Seung Min Kim

Cheol Keun Kim

Dong In Jo

Soon Heum Kim

https://orcid.org/0000-0002-5021-4205

https://orcid.org/0000-0003-2310-2125

https://orcid.org/0000-0003-2126-9749

https://orcid.org/0000-0002-3075-4482

https://orcid.org/0000-0001-9773-4753

\section{Supplemental material}

Supplemental Digital Content 1. Mixing of purified fat with red paint diluted to the density of platelet-rich plasma. 
Supplemental data can be found at: https://doi.org/10.14730/aaps. 2019.01788

\section{REFERENCES}

1. Neuber F. Fettransplantation. Chir Kongr Verhandl Dsch Gesellch Chir 1893;22:66.

2. Coleman SR. Facial recontouring with lipostructure. Clin Plast Surg 1997;24:347-67.

3. Tolba AM, Nasr M. Initial experience of face augmentation using fat graft-platlet rich plasma mix. Surg Sci 2015;6:489-98.

4. Nguyen A, Pasyk KA, Bouvier TN, et al. Comparative study of survival of autologous adipose tissue taken and transplanted by different techniques. Plast Reconstr Surg 1990;85:378-86.

5. Matsudo PK, Toledo LS. Experience of injected fat grafting. Aesthetic Plast Surg 1988;12:35-8.

6. Moore JH Jr, Kolaczynski JW, Morales LM, et al. Viability of fat obtained by syringe suction lipectomy: effects of local anesthesia with lidocaine. Aesthetic Plast Surg 1995;19:335-9.

7. Shiffman MA, Mirrafati S. Fat transfer techniques: the effect of harvest and transfer methods on adipocyte viability and review of the literature. Dermatol Surg 2001;27:819-26.

8. Cervelli V, De Angelis B, Lucarini L, et al. Tissue regeneration in loss of substance on the lower limbs through use of platelet-rich plasma, stem cells from adipose tissue, and hyaluronic acid. Adv Skin Wound Care 2010;23:262-72.

9. Li F, Guo W, Li K, et al. Improved fat graft survival by different volume fractions of platelet-rich plasma and adipose-derived stem cells. Aesthet Surg J 2015;35:319-33.

10. Modarressi A. Platlet rich plasma (PRP) improves fat grafting outcomes. World J Plast Surg 2013;2:6-13.

11. Hunstad JP. Tumescent and syringe liposculpture: a logical partnership. Aesthetic Plast Surg 1995;19:321-33.

12. Topcu A, Aydin OE, Unlu M, et al. Increasing the viability of fat grafts by vascular endothelial growth factor. Arch Facial Plast Surg 2012;14: 270-6.

13. Yuksel E, Weinfeld AB, Cleek R, et al. Increased free fat-graft survival with the long-term, local delivery of insulin, insulin-like growth factor-I, and basic fibroblast growth factor by PLGA/PEG microspheres. Plast Reconstr Surg 2000;105:1712-20.

14. Cheriyan T, Kao HK, Qiao X, et al. Low harvest pressure enhances autologous fat graft viability. Plast Reconstr Surg 2014;133:1365-8.

15. Fangrow T. Syringe pressure calculator [Internet]. Tom Fangrow [cited 2019 Sep 11]. Available from: http://tomfangrow.com/Syringe $\% 20$ Pressure.html. 